\title{
Correlaciones entre Marcadores de Grasa Abdominal Obteni- dos por Densitometría y Técnicas Antropométricas Convencio- nales en Mujeres Postmenopausicas con Síndrome Metabólico
}

\author{
Correlations between Markers of Abdominal Fat Obtained by DXA and Anthropometric \\ Conventional Techniques in Postmenopausal Women with Metabolic Syndrome
}

\author{
Rosety-Rodriguez, M.*; Tejerina, A.**; Camacho-Molina, A..**; Rosety, I.****; Diaz, A. J."; Fornieles, G..; \\ Rodriguez-Pareja, A. ${ }^{* * * * *}$; Rosety, M. A. ${ }^{* * * * *}$ Alvero-Cruz, J. R. ${ }^{* * * * * *}$ \& Ordonez, F. J. ${ }^{* * * *}$
}

ROSETY-RODRIGUEZ, M.; TEJERINA, A.; CAMACHO-MOLINA, A.; ROSETY, I.; DIAZ, A. J.; FORNIELES, G.; RODRIGUEZ-PAREJA, A.; ROSETY, M. A.; ALVERO-CRUZ, J. R. \& ORDONEZ, F. J. Correlaciones entre marcadores de grasa abdominal obtenidos por densitometría y técnicas antropométricas convencionales en mujeres postmenopausicas con síndrome metabólico. Int. J. Morphol., 31(4):1415-1420, 2013.

RESUMEN: El presente estudio pretende identificar correlaciones estadísticamente significativas entre parámetros de masa grasa abdominal obtenidos por densitometría (DXA) y otros de tipo cineantropométrico (índices de distribución de masa grasa) y bioquímico (perfil lipídico) en mujeres postmenopausicas con síndrome metabólico. Se diseño un estudio de cohortes histórico que incluyó a un total de 1326 mujeres post-menopausicas con edad > 45 años que se habían sometido rutinariamente a DXA para conocer su densidad mineral ósea entre Enero de 2006 y Enero de 2011. Se utilizó un DXA tipo Lunar DPX-L para determinar la masa grasa abdominal en las regiones de interés L1-L4 y L3-L4. Además del DXA, se obtuvo de cada participante la correspondiente anamnesis, bioquímica, tensión arterial e índices de distribución de masa grasa mediante técnicas antropométricas convencionales. Se utilizó la clasificación NCEP-ATP-III para el diagnóstico de síndrome metabólico. Este protocolo fue aprobado por un Comité de Ética Institucional. La mayor fuerza de asociación se estableció entre el porcentaje de masa grasa L1-L4 obtenido por DXA y el perímetro de la cintura $(r=0,77 ; \mathrm{p}=0,0016)$ además de con colesterol-HDL ( $r=-0,58 ; \mathrm{p}=0,0290)$. Finalmente se concluye que el perímetro de la cintura y los niveles de colesterol-HDL podrían recomendarse como predictores del comportamiento de la masa grasa abdominal de regiones de interés L1-L4 y L3-L4 obtenidas por DXA en mujeres postmenopausicas con síndrome metabólico.

PALABRAS CLAVE: Síndrome metabólico; Obesidad abdominal; Postmenopausia; Absorciometría.

\section{INTRODUCCIÓN}

La prevalencia de síndrome metabólico es cada vez mayor entre mujeres postmenopausicas no solo en España (Llisterri et al., 2009) sino también en Latinoamérica (Royer et al., 2007). Aunque en la actualidad existen diferentes clasificaciones diagnósticas, todas coinciden en reconocer la importancia de la obesidad abdominal o central en el origen y progresión del síndrome metabólico (Després et al., 2008). En este sentido, los adipocitos de la grasa abdominal o central o visceral jugarían un papel más importante que la subcutánea en la secreción de citokinas proinflamatorias (adipokinas) como demuestran los mayores niveles de ARNm presentes en aquellos (Després et al.; Philips \& Prins, 2008).
En los últimos años ha aumentado el interés por estudiar el comportamiento de la masa grasa abdominal en el manejo clínico de esto pacientes (Kishida et al., 2012; Philips et al., 2013). Las técnicas cineantropométricas convencionales siguen siendo usadas gracias a su bajo coste y disponibilidad en cualquier entorno clínico (Oda et al., 2010). Sin embargo este tipo de herramientas presenta limitaciones como no poder diferenciar masa grasa visceral de la subcutánea a pesar de su diferente rol en la fisiopatología del síndrome metabólico (Bouchard, 2007). También ofrece una limitada capacidad para reconocer cambios en los niveles de masa grasa atribuidos a programas de intervención basa-

\footnotetext{
Departamento de Medicina. Facultad de Medicina, Universidad de Cadiz, Cadiz, España.

** Servicio de Radiología. Hospital Jiménez Díaz, Universidad Autónoma de Madrid, Madrid, España.

**** Servicio de Medicina Interna. Hospital Juan Ramón Jiménez, Huelva, España.

**** Departamento de Anatomía y Embriología Humana. Facultad de Medicina, Universidad de Cadiz, Cadiz, España.

****** Servicio de Ginecología. Hospital Universitario de Jerez, Cadiz, España.

****** Grupo Español de Cine antropometría. Facultad de Medicina. Universidad de Malaga, Malaga, España.
} 
ROSETY-RODRIGUEZ, M.; TEJERINA, A.; CAMACHO-MOLINA, A.; ROSETY, I.; DIAZ, A. J.; FORNIELES, G.; RODRIGUEZ-PAREJA, A.; ROSETY, M. A.; ALVERO-CRUZ, J. R. \& ORDONEZ, F. J. Correlaciones entre marcadores de grasa abdominal obtenidos por densitometría y técnicas antropométricas convencionales en mujeres postmenopausicas con síndrome metabólico. Int. J. Morphol., 31(4):1415-1420, 2013.

dos en dietas hipocalóricas, ejercicio, etc. En este sentido, cada vez son más los estudios que recomiendan el uso de pruebas de imagen por ser más precisas y reproducibles. La reciente incorporación de las regiones de interés (ROI) en el software del DXA (absorciometría de rayos X de energía dual) está haciendo que esta herramienta resulte cada vez más atractiva para conocer la masa grasa abdominal tanto a nivel clínico como investigador (Direk et al., 2013; Micklesfield et al., 2012). Todo ello resulta de especial interés en mujeres post-menopáusicas ya que éstas participan regularmente en screenings contra la osteoporosis mediante DXA (Powell et al., 2012) con el ahorro adicional que ello supondría.

El presente estudio pretende identificar correlaciones estadísticamente significativas entre parámetros de masa grasa abdominal obtenidos por DXA y otros de tipo cineantropométrico (índices de distribución de masa grasa) y bioquímica (perfil lipídico) en mujeres postmenopausicas con síndrome metabólico. La utilidad práctica de este estudio sería contribuir al diagnóstico precoz de dicho proceso mediante el uso de herramientas menos costosas, complejas e invasivas.

\section{MATERIAL Y METODO}

Población muestral. En nuestro estudio de cohortes histórico o retrospectivo se incluyeron a un total de $1326 \mathrm{mu}$ jeres post-menopausicas con edad > 45 años que se habían sometido rutinariamente a DXA para conocer su densidad mineral ósea entre Enero de 2006 y Enero de 2011.

Como criterios de inclusión estaban previstos: ser mujer; post-menopaúsica; edad > 45 años en el momento de someterse al DXA. De existir varios registros DXA para una misma paciente, se recurrió al más antiguo de ellos.

Como criterios de exclusión se contempló la coexistencia de: patologías del tiroides no corregidas por su impacto en la composición corporal de estos pacientes; diabetes; patología cardiovascular isquémica; artritis reumatoide; EPOC; demencia; tratamiento con corticosteroides de mas de 90 días durante el año previo al examen con DXA. El diagnóstico clínico de síndrome metabólico, fue de acuerdo con el National Cholesterol Education Program Adult Treatment Panel III (ATP-III) (Grundy et al., 2005).

Los diferentes parámetros antropométricos, bioquímicos y clínicos estudiados se obtuvieron de la revisión de los registros de las pacientes.
Variables de laboratorio. Para el estudio de las variables séricas de las participantes se extrajo una muestra de sangre venosa antecubital por la mañana tras un ayuno de 10-12 h, cumpliendo en todo momento con los principios establecidos en la Declaración de Helsinki de la Asociación Médica Mundial (2004). Tras recogerse en un tubo (EDTA) se centrifugó durante 10 minutos (1000 rpm) obteniendo alícuotas de suero que se almacenaron hasta su análisis en un ultracongelador vertical a $-70^{\circ} \mathrm{C}$.

Concretamente, por procedimientos habituales de laboratorio se determinaron el perfil lipídico sérico (colesterolLDL; colesterol-HDL; ratiocolesterol-total/colesterol-HDL; triglicéridos) y la glucemia basal de las participantes.

Variables cineantropométricas. Se utilizó un DXA tipo Lunar DPX-L (Lunar Co, Wisconsin, USA). Este aparato ha sido ampliamente utilizado en la literatura por presentar un software que facilita enormemente al radiólogo establecer regiones de interés (ROI) para el estudio de la masa grasa corporal. Precisamente las regiones de interés previstas en nuestro trabajo serán la zona lumbar L1-L4 y la zona L3L4. A todo ello habrá que añadir parámetros estándar que facilita el software como es el porcentaje de masa grasa del tronco.

Merece ser enfatizado que el tronco incluye el propio tronco y el abdomen, excluyendo la pelvis. Con este procedimiento los sujetos se exponen a una radiación mínima de entre 0,015-0,06 mrem dependiendo del diámetro antero-posterior de la persona objeto de estudio que equivale a entre el 1-10\% de una radiografía simple de tórax.

En cuanto a los índices de distribución de la masa grasa se evaluaron: perímetro de la cintura, perímetro de la cadera e índice cintura/cadera. Las mediciones de cintura y cadera se harán con el sujeto en bipedestación siendo necesaria la participación de un colaborador para mantener la cinta por el lado opuesto al de la lectura. Finalmente el índice de masa corporal se determinará de acuerdo con la ecuación: IMC = peso $(\mathrm{kg}) /$ talla $(\mathrm{m}) 2$. Para tal fin utilizaremos tallímetro, balanza digital y cinta antropométrica (Holtain Ltd).

Presión arterial. La tensión arterial sistólica (TAS) y diastólica (TAD) se midió mediante esfigomanómetro (modelo ALPK2) tras 5-10 minutos de reposo en sedestación.

Aspectos éticos y estadísticos. Se respetó la Declaración de Helsinki (2002). Asimismo nuestro grupo diseñó un modelo de consentimiento informado para ser firmado por cada participante con la que se contactó previamente por vía telefónica y a la que se le envió por correo prefranqueado. Por todo ello merece ser enfatizado que nuestro protocolo fue 
ROSETY-RODRIGUEZ, M.; TEJERINA, A.; CAMACHO-MOLINA, A.; ROSETY, I.; DIAZ, A. J.; FORNIELES, G.; RODRIGUEZ-PAREJA, A.; ROSETY, M. A.; ALVERO-CRUZ, J. R. \& ORDONEZ, F. J. Correlaciones entre marcadores de grasa abdominal obtenidos por densitometría y técnicas antropométricas convencionales en mujeres postmenopausicas con síndrome metabólico. Int. J. Morphol., 31(4):1415-1420, 2013.

aprobado por un Comité de Ética Institucional (Universidad de Sevilla).

Los resultados se presentaron como media, desviación estándar (DE) e intervalo de confianza al 95\% (IC95\%). Centrándonos más concretamente en el grupo de mujeres postmenopáusicas con síndrome metabólico, se identificaron correlaciones entre la masa grasa obtenida por DXA y otras variables cineantropométricas y bioquímicas ensayadas mediante el coeficiente de correlación " $r$ " de Pearson.

\section{RESULTADOS}

Las características antropométricas, bioquímicas y clínicas de la cohorte histórica objeto de estudio se recogen en la Tabla I. Del total de dicha cohorte, el 40,5\% $(n=537)$ cumplió los criterios diagnósticos de síndrome metabólico previstos en la clasificación ATP-III. Sus características se detallan en la Tabla II. Precisamente, en el grupo de mujeres postmenopausicas con diagnóstico de síndrome metabólicos se han puesto de manifiesto correlaciones estadísticamente significativas entre marcadores de masa grasa abdominal obtenidos por DXA y por métodos cineantropométricos convencionales. La mayor fuerza de asociación se ha establecido entre la masa grasa de la región L1-L4 y el perímetro de la cintura $(r=0,77 ; p=0,0016)$. Todos estos valores se enumeran en la Tabla III. De igual modo, en este mismo grupo,

Tabla I. Características antropométricas, bioquímicas y clínicas de las mujeres postmenopausicas incluidas en el estudio de cohortes histórico (n=1326).

\begin{tabular}{lccc}
\hline & Media & $\mathbf{\pm D E}$ & IC al 95\% \\
\hline Edad (años) & 52,1 & $\pm 6,5$ & $46,0-59,8$ \\
IMC (kg/m²) & 28,9 & $\pm 4,1$ & $26,3-30,1$ \\
PC (cm) & 89,8 & $\pm 4,6$ & $86,7-93,4$ \\
ICC & 0,83 & $\pm 0,09$ & $0,79-0,85$ \\
DXA & & & \\
Masa grasa total (\%) & 30,8 & $\pm 5,5$ & $29,1-32,3$ \\
Masa grasa tronco(\%) & 26,0 & $\pm 5,8$ & $22,8-27,9$ \\
Masa grasa L1-L4 & 32,2 & $\pm 5,2$ & $30,5-35,1$ \\
Masa grasa L4-L5 & 33,8 & $\pm 5,6$ & $31,4-37,1$ \\
c-LDL (mg/dl) & 108,4 & $\pm 15,1$ & $91,7-132,4$ \\
c-HDL (mg/dl) & 59,2 & $\pm 7,1$ & $51,5-68,7$ \\
Triglicéridos (mg/dl) & 131,8 & $\pm 16,4$ & $118,6-144,2$ \\
Glucemia (mg/dl) & 94,7 & $\pm 8,3$ & $88,2-100,5$ \\
TAS (mm Hg) & 122,6 & $\pm 12,6$ & $116,4-128,0$ \\
TAD (mm Hg) & 75,8 & $\pm 9,3$ & $70,7-81,6$ \\
\hline
\end{tabular}

$\mathrm{PC}=$ Perímetro de la cintura, $\mathrm{ICC}=$ Índice cintura/cadera, $\mathrm{DXA}=$ Absorciometría de rayos X de energía dual, c-LDL= colesterolLDL, c-HDL= colesterol-HDL, TAS= Tensión arterial sistólica, $\mathrm{TAD}=$ Tensión arterial diastólica. se identificaron correlaciones significativas entre marcadores obtenidos por DXA y el perfil lipídico sérico. La mayor fuerza de asociación, aunque de naturaleza negativa, se estableció entre la masa grasa L1-L4 y los niveles de colesterol HDL ( $\mathrm{r}=-0,58 ; \mathrm{p}=0,0290)$. Todos estos datos se resumen en la Tabla IV.

Tabla II. Características antropométricas, bioquímicas y clínicas de las mujeres postmenopausicas incluidas en el estudio de cohortes histórico que cumplían los criterios diagnósticos ATP-III de syndrome metabólico $(\mathrm{n}=537)$.

\begin{tabular}{lccc}
\hline & Media & $\mathbf{\pm D E}$ & IC al 95\% \\
\hline Edad (años) & 53,7 & $\pm 4,4$ & $48,2-60,4$ \\
IMC (kg/m²) & 33,8 & $\pm 3,8$ & $29,4-35,9$ \\
PC (cm) & 100,2 & $\pm 4,1$ & $94,2-104,3$ \\
ICC & 0,86 & $\pm 0,07$ & $0,83-0,91$ \\
DXA & & & \\
masa grasa total (\%) & 37,0 & $\pm 3,9$ & $34,8-38,6$ \\
masa grasa tronco(\%) & 30,6 & $\pm 3,7$ & $28,9-32,0$ \\
masa grasa L1-L4 (\%) & 39,3 & $\pm 3,8$ & $37,8-40,7$ \\
masa grasa L4-L5 (\%) & 41,2 & $\pm 4,0$ & $38,6-42,9$ \\
c-LDL (mg/dl) & 132,2 & $\pm 14,4$ & $110,4-156,8$ \\
c-HDL (mg/dl) & 47,7 & $\pm 6,8$ & $42,4-53,2$ \\
Triglicéridos (mg/dl) & 170,9 & $\pm 15,1$ & $126,3-201,7$ \\
Glucemia (mg/dl) & 112,6 & $\pm 8,7$ & $98,2-125,8$ \\
TAS (mm Hg) & 138,6 & $\pm 11,9$ & $126,2-150,1$ \\
TAD (mm Hg) & 86,8 & $\pm 8,8$ & $76,4-95,2$ \\
\hline
\end{tabular}

$\mathrm{PC}=$ Perímetro de la cintura, $\mathrm{ICC}=$ Índice cintura/cadera, $\mathrm{DXA}=$ Absorciometría de rayos $\mathrm{X}$ de energía dual, c-LDL $=$ colesterol-LDL, c$\mathrm{HDL}=$ colesterol-HDL, TAS $=$ Tensión arterial sistólica, $\mathrm{TAD}=$ Tensión arterial diastólica.

Tabla III. Correlación ("r" de Pearson) entre marcadores de masa grasa abdominal obtenidos por densitometría (DXA) y por métodos cineantropométricos convencionales en mujeres postmenopáusicas con síndrome metabólico.

\begin{tabular}{lccc}
\hline & IMC & PC & ICC \\
\hline Grasa tronco & $0,58^{*}$ & $0,66^{* *}$ & $0,63^{*}$ \\
Grasa L1-L4 & $0,63^{*}$ & $0,77^{* *}$ & $0,70^{*}$ \\
Grasa L3-14 & $0,61^{*}$ & $0,75^{* *}$ & $0,71^{* *}$ \\
\hline
\end{tabular}

$\mathrm{IMC}=$ Índice masa corporal, $\mathrm{PC}=$ Perímetro cintura, $\mathrm{ICC}=$ Índice cintura/ cadera, $*=$ Significativo con $\mathrm{p}<0,05, * *=$ Significativo $\operatorname{con} \mathrm{p}<0,001$.

\section{DISCUSIÓN}

Nuestros resultados han confirmado la existencia de una fuerte correlación entre marcadores de masa grasa abdominal obtenidos por DXA y aquellos obtenidos por técnicas antropométricas convencionales así como con el perfil lipídico de mujeres postmenopausicas con síndrome metabólico. Resultados similares fueron publicados en mujeres premenopausicas (Hwang et al., 2008) y postmenopáusicas (Vatanparast et al., 2009) sin diagnóstico de 
ROSETY-RODRIGUEZ, M.; TEJERINA, A.; CAMACHO-MOLINA, A.; ROSETY, I.; DIAZ, A. J.; FORNIELES, G.; RODRIGUEZ-PAREJA, A.; ROSETY, M. A.; ALVERO-CRUZ, J. R. \& ORDONEZ, F. J. Correlaciones entre marcadores de grasa abdominal obtenidos por densitometría y técnicas antropométricas convencionales en mujeres postmenopausicas con síndrome metabólico. Int. J. Morphol., 31(4):1415-1420, 2013

síndrome metabólico. La identificación de estas correlaciones resulta de especial interés porque por su elevado coste y la necesidad de contar con personal cualificado, el DXA no está al alcance de cualquier entorno clínico. Por el contrario, las técnicas antropométricas convencionales y la bioquímica básica si son más accesibles a nivel médicoasistencial, por lo que podrían servirnos como predictores del comportamiento de la masa grasa abdominal habida cuenta que ésta ha sido propuesta como diana terapéutica en pacientes con SM (Kishida et al.).

Paralelamente, el creciente interés por la obesidad abdominal o central entre los pacientes con síndrome metabólico (Després et al.), justifica la menor importancia que recibe la grasa corporal total, que incluye también a la subcutánea, lo que podría llevar a errores diagnósticos y pronósticos. Esto hace, que se subestimen porcentajes de grasa abdominal visceral e incluso los diagnósticos de síndrome metabólico, sobre todo en mujeres debido a las diferencias entre los modelos androide y ginecoide de distribución de masa grasa (von Eyben et al., 2003).

De manera más detallada, individuos ajustados en masa grasa subcutánea, mostraban una mayor o menor resistencia a la insulina en función de su mayor o menor depósito graso visceral o abdominal. Por el contrario, sujetos ajustados en lo que respecta a la masa grasa abdominal mostraban la misma sensibilidad a la insulina con independencia del mayor o menor acúmulo de grasa subcutánea (Ross et al., 2002).

Resultados similares han sido referidos por Leslie $e t$ al. (2010) en una cohorte de 30252 mujeres mayores de 40 años. En concreto, el riesgo de desarrollar diabetes tipo II en esta cohorte era 3,56 veces mayor entre aquellas que presentaban más masa grasa abdominal cuando se comparan las que se encontraban en el quintil menor, ajustadas en edad, índice de masa corporal y comorbilidad.

Tal y como habían referido estudios previos (Royer et al.; Llisterri et al.), nuestros resultados vuelven a confirmar que la prevalencia de síndrome metabólico en mujeres postmenopausicas es muy preocupante. Este hecho podría explicarse por la pérdida del efecto protector cardiovascular de los estrógenos, existe un mayor acumulo de grasa visceral o abdominal que es la que se ha asociado a resistencia a la insulina, hipertensión y al propio síndrome metabólico (Marjani \& Moghasemi, 2012). De hecho, la mortalidad por origen cardiovascular sigue siendo significativamente mayor en este grupo cuando se compara con controles no menopausicas (Rosamond et al., 2007). Habernos centrado en el estudio de mujeres postmenopáusicas también estaría justificado por ser las que se realizan densitometrías con mayor frecuencia para descartar pérdidas de densidad mineral ósea. Sin embargo esta misma podría ser una limitación del estudio ya que solo se han seleccionado mujeres sometidas al screening de osteoporosis, por lo que estos resultados deben extrapolarse a población general con cautela. Con todo merece ser puntualizado que la presencia de síndrome metabólico no va a empeorar los niveles de densidad mineral ósea en mujeres post-menopáusicas. De hecho parece existir un equilibrio entre factores que reducen la absorción mineral ósea (p. ej. obesidad) y otros que la aumentan (estatus proinflamatorio; hipertensión arterial) (Jeon et al., 2011).

En el otro extremo, merece ser destacado que una de las principales fortalezas de nuestro trabajo es el tamaño de la cohorte estudiada lo que contribuirá a reforzar los resultados obtenidos en nuestro entorno. Finalmente se concluye que el perímetro de la cintura y los niveles de colesterolHDL podrían recomendarse como predictores del comportamiento de la masa grasa abdominal de regiones de interés L1-L4 y L3-L4 obtenidas por DXA en mujeres postmenopausicas con síndrome metabólico. La utilidad práctica sería contribuir al diagnóstico precoz que a su vez permitiría introducir cambios de estilos de vida más saludables, así como tratamientos preventivos que reduzcan la morbi-mortalidad y costes sanitarios asociados a dicho proceso. Futuros estudios son aún necesarios para consolidar esta línea de trabajo no solo en el ámbito de la investigación sino también en el de su aplicación clínica.

ROSETY-RODRIGUEZ, M.; TEJERINA, A.; CAMACHOMOLINA, A.; ROSETY, I.; DIAZ, A. J.; FORNIELES, G.; RODRIGUEZ-PAREJA, A.; ROSETY, M. A.; ALVEROCRUZ, J. R. \& ORDONEZ, F. J. Correlations between markers of abdominal fat obtained by DXA and anthropometric conventional techniques in postmenopausal women with metabolic syndrome. Int. J. Morphol., 31(4):1415-1420, 2013.

SUMMARY: The current study was conducted to identify potential correlations between abdominal fat mass obtained by DXA and several parameters obtained by anthropometric conventional techniques as well as lipid profile in postmenopausal women with MS. This historical cohort study included a total of 1326 postmenopausal women aged $>45$ years old who had routinely undergone DXA to measure their bone mineral density between January 2006 and January 2011. The regions of interest envisaged in our study by using DXA were the lumbar regions L1-L4 and L4-L5. At the same time, they underwent a complete medical examination including personal medical history assessment, biochemical blood analysis, blood pressure measurements and anthropometrical evaluation. Metabolic syndrome was diagnosed attending to the criteria established by National Cholesterol Education Program Adult 
ROSETY-RODRIGUEZ, M.; TEJERINA, A.; CAMACHO-MOLINA, A.; ROSETY, I.; DIAZ, A. J.; FORNIELES, G.; RODRIGUEZ-PAREJA, A.; ROSETY, M. A.; ALVERO-CRUZ, J. R. \& ORDONEZ, F. J. Correlaciones entre marcadores de grasa abdominal obtenidos por densitometría y técnicas antropométricas convencionales en mujeres postmenopausicas con síndrome metabólico. Int. J. Morphol., 31(4):1415-1420, 2013.

Treatment Panel III (NECP-ATP-III). This protocol was approved by an Institutional Ethics Committee. Several significant correlations were found between DXA and indices of body fat distribution as well as lipid profile. The strongest correlations were found between fat mass L1-L4 and waist circumference $(\mathrm{r}=0.77 ; \mathrm{p}=0.0016)$ and levels of HDL-cholesterol $(\mathrm{r}=-0.58$; $\mathrm{p}=0.0290$ ). It was concluded that waist circumference and HDLcholesterol may be recommended to predict fat mass in regions of interest L1-L4 and L3-L4 in postmenopausal women with MS.

KEY WORDS: Metabolic syndrome; Postmenopausal; Abdominal obesity; Absorptiometry.

\section{REFERENCIAS BIBLIOGRÁFICAS}

Bouchard, C. BMI, fat mass, abdominal adiposity and visceral fat: where is the 'beef'. Int. J. Obes. (Lond.), 31(10):1552-3, 2007.

Després, J. P.; Lemieux, I.; Bergeron, J.; Pibarot, P.; Mathieu, P.; Larose, E.; Rodés-Cabau, J.; Bertrand, O. F. \& Poirier, P. Abdominal obesity and the metabolic syndrome: contribution to global cardiometabolic risk. Arterioscler. Thromb. Vasc. Biol., 28(6):1039-49, 2008.

Direk, K.; Cecelja, M.; Astle, W.; Chowienczyk, P.; Spector, T. D.; Falchi, M \& Andrew, T. The relationship between DXA-based and anthropometric measures of visceral fat and morbidity in women. BMC Cardiovasc. Disord., 13:25, 2013.

Grundy, S. M.; Cleeman, J. I.; Daniel, S. R.; Donato, K. A.; Eckel, R. H.; Franklin, B. A.; Gordon, D. J.; Krauss, R. M.; Savage, P. J.; Smith, S. C. Jr.; Spertus, J. A.; Costa, F.; American Heart Association \& National Heart, Lung, and Blood Institute. Diagnosis and management of the metabolic syndrome: an American Heart Association/ National Heart, Lung, and Blood Institute scientific statement. Circulation, 112(17):2735-52, 2005.

Hwang, M. J.; Chung, W. S.; Gallagher, D.; Kim, D. Y.; Shin, H. D. \& Song, M. Y. How useful is waist circumference for assessment of abdominal obesity in Korean pre-menopausal women during weight loss? Asia. Pac. J. Clin. Nutr., 17(2):229-34, 2008.

Jeon, Y. K.; Lee, J. G.; Kim, S. S.; Kim, B. H.; Kim, S. J.; Kim, Y. K. \& Kim, I. J. Association between bone mineral density and metabolic syndrome in pre- and postmenopausal women. Endocr. J., 58(2):87-93, 2011.
Kishida, K.; Funahashi, T.; Matsuzawa, Y. \& Shimomura, I. Visceral adiposity as a target for the management of the metabolic syndrome. Ann. Med., 44(3):233-41, 2012.

Leslie, W. D.; Ludwig, S. M. \& Morin, S. Abdominal fat from spine dual energy X-ray absorptiometry and risk for subsequent diabetes. J. Clin. Endocrinol. Metab., 95(7):3272-6, 2010.

Llisterri, J. L.; Cea-Calvo, L.; Martí-Canales, J. C.; Lozano, J. V.; Aznar, J. \& Redón, J. Prevalence of metabolic syndrome in Spanish population aged 60 years-old or more. PREV-ICTUS, a population-based study. Med. Clin. (Barc.), 132(5):172-9, 2009.

Marjani, A. \& Moghasemi, S. The Metabolic Syndrome among Postmenopausal Women in Gorgan. Int. J. Endocrinol., 2012:953627, 2012.

Micklesfield, L. K.; Goedecke, J. H.; Punyanitya, M.; Wilson, K. E. \& Kelly, T. L. Dual-energy X-ray performs as well as clinical computed tomography for the measurement of visceral fat. Obesity (Silver Spring), 20(5):1109-14, 2012.

Oda, E. Optimal cutoff points of waist circumference for the criteria of abdominal obesity: comparison with the criteria of the International Diabetes Federation. Circ. J., 74(1):207, 2010.

Phillips, L. K. \& Prins, J. B. The link between abdominal obesity and the metabolic syndrome. Curr. Hypertens. Rep., 10(2):156-64, 2008.

Phillips, C. M.; Tierney, A. C.; Perez-Martinez, P.; Defoort, C.; Blaak, E. E.; Gjelstad, I. M.; Lopez-Miranda, J.; KiecKlimczak, M.; Malczewska-Malec, M.; Drevon, C. A.; Hall, W.; Lovegrove, J. A.; Karlstrom, B.; Risérus, U. \& Roche, H. M. Obesity and body fat classification in the metabolic syndrome: Impact on cardiometabolic risk metabotype. Obesity (Silver Spring), 21(1):E154-61, 2013.

Powell, H.; O'Connor, K. \& Greenberg, D. Adherence to the U.S. Preventive Services Task Force 2002 osteoporosis screening guidelines in academic primary care settings. J. Womens Health (Larchmt), 21(1):50-3, 2012.

Rosamond, W.; Flegal, K.; Friday, G.; Go, A.; Greenlund, K.; Haase, N.; Ho, M.; Howard, V.; Kissela, B.; Kittner, S.; Lloyd-Jones, D.; McDermott, M.; Meigs, J.; Moy, C.; Nichol, G.; O'Donnell, C. J.; Roger, V.; Rumsfeld, 
ROSETY-RODRIGUEZ, M.; TEJERINA, A.; CAMACHO-MOLINA, A.; ROSETY, I.; DIAZ, A. J.; FORNIELES, G.; RODRIGUEZ-PAREJA, A.; ROSETY, M. A.; ALVERO-CRUZ, J. R. \& ORDONEZ, F. J. Correlaciones entre marcadores de grasa abdominal obtenidos por densitometría y técnicas antropométricas convencionales en mujeres postmenopausicas con síndrome metabólico. Int. J. Morphol., 31(4):1415-1420, 2013.

J.; Sorlie, P.; Steinberger, J.; Thom, T.; WasserthielSmoller, S.; Hong, Y. \& American Heart Association Statistics Committee and Stroke Statistics Subcommittee. Heart disease and stroke statistics—2007 Update: a report from the American Heart Association Statistics Committee and Stroke Statistics Subcommittee. Circulation, 115(5):e69-171, 2007.

Ross, R.; Aru, J.; Freeman, J.; Hudson, R. \& Cansen, I. Abdominal adiposity and insulin resistance in obese men. Am. J. Physiol. Endocrinol. Metab., 282(3):E657-63, 2002.

Royer, M.; Castelo-Branco, C.; Blümel, J. E.; Chedraui, P. A.; Danckers, L.; Bencosme, A.; Navarro, D.; Vallejo, S.; Espinoza, M. T.; Gómez, G.; Izaguirre, H.; Ayala, F.; Martino, M.; Ojeda, E.; Onatra, W.; Saavedra, J.; Tserotas, K.; Pozzo, E.; Manriquez, V.; Prada, M.; Grandia, E.; Zuniga, C.; Lange, D.; Sayegh, F. \& Collaborative Group for Research of the Climacteric in Latin America. The US National Cholesterol Education Programme Adult Treatment Panel III (NCEP ATP III): prevalence of the metabolic syndrome in postmenopausal Latin American women. Climacteric, 10(2):164-70, 2007.

Vatanparast, H.; Chilibeck, P. D.; Cornish, S. M.; Little, J. P.; Paus-Jenssen, L. S.; Case, A. M. \& Biem, H. J. DXAderived abdominal fat mass, waist circumference, and blood lipids in postmenopausal women. Obesity (Silver Spring), 17(8):1635-40, 2009.

von Eyben, F. E.; Mouritsen, E.; Holm, J.; Montvilas, P.; Dimcevski, G.; Suciu, G.; Helleberg, I.; Kristensen, L. \& von Eyben, R. Intra-abdominal obesity and metabolic risk factors: a study of young adults. Int. J. Obes. Relat. Metab. Disord., 27(8):941-9, 2003.

\section{Direccción para Correspondencia: Francisco J. Ordonez. MD, PhD. Human Anatomy Department School of Medicine University of Cadiz Pza. Fragela s/n 11003 Cadiz ESPAÑA}

Email: franciscojavier.ordonez@uca.es

Recibido : 09-08-2013

Aceptado: 12-10-2013 